\title{
Thiamin status in normal and malnourished children in Jamaica
}

\author{
By B. HAILEMARIAM, J. P. LANDMAN AND ALAN A. JACKSON* \\ Tropical Metabolism Research Unit, University of the West Indies, Mona, \\ Kingston 7, Jamaica
}

\section{(Received 21 February 1984 - Accepted 15 November 1984)}

\footnotetext{
1. Thiamin status has been measured using the erythrocyte transketolase $(E C 2.2 .1 .1)$ assay in twenty-eight normal children and in twenty-five severely-malnourished children throughout the course of recovery.

2. Subclinical thiamin deficiency was found in $7 \%$ of the normal children and $36 \%$ of the malnourished children on admission.

3. There was no significant association between thiamin status and oedema, stunting or wasting, history of breast-feeding, pattern of weaning, age or sex.

4. Five malnourished children, who died, all had a normal thiamin status on admission; however, two developed biochemical evidence of thiamin deficiency preterminally.
}

Severe malnutrition in childhood presents a wide spectrum of clinical features, representing the complex interplay of specific nutrient deficiencies (Landman \& Jackson, 1980). Although thiamin deficiency has been recognized as playing an important role in specific deficiencies in certain environments, its relation to childhood malnutrition has not been particularly noted.

It had been our clinical impression that some of the children being admitted to our ward with severe malnutrition presented signs that could be attributed to thiamin deficiency, and they showed a good therapeutic response to thiamin supplementation. A retrospective analysis covering 5 years from 1978 revealed that eleven of 314 admissions $(3.5 \%)$ may have developed thiamin deficiency at some time after admission. In the four most dramatic examples, high output cardiac failure was associated with complete anuria and increasing oedema. The response to parenteral thiamin was not necessarily specific but urine flow was established within $6 \mathrm{~h}$. Patrick (1977) described a syndrome in which a small group of children developed congestive heart failure during the early phase of rehabilitation, at a time when the energy intake was in excess of maintenance requirements. We wondered if this represented a relative thiamin deficiency, provoked by the stress of an increased energy intake.

Extensive assessments of thiamin nutriture have been conducted by the Interdepartmental Committee on Nutrition for National Defense (ICNND) on normal populations in Asia (ICNND, 1963a), Africa (ICNND 1963b), Latin America (ICNND, 1960) and the eastern Caribbean (ICNND, 1962). Little information is available on children with severe malnutrition. In Guatemala (Scrimshaw \& Behar, 1961), malnourished children were found to have normal levels of thiamin in serum whereas, in northern Thailand, a reduced excretion of thiamin in urine $(24 \mathrm{~h})$ suggested the presence of thiamin deficiency in up to $9 \%$ of children (Thanangkul, 1975). Erythrocyte transketolase (EC 2.2.1.1) activity (ETKA) has been used to demonstrate biochemical evidence of thiamin deficiency in normal and malnourished Ghanaian children (Neumann et al. 1979) and in malnourished children in Nigeria (Laditan \& Ette, 1983).

The use of different methods to measure thiamin status by different investigators makes it difficult to draw comparisons from one study to another. Brin et al. (1958) considered 
the measurement of ETKA as the most specific method for assessing the functional state at a tissue level. We have assessed thiamin status using ETKA in children admitted to hospital with severe malnutrition and compared the results with healthy children of the same age. Our findings indicate that thiamin deficiency in children is less common than previously reported for children in the Caribbean (ICNND, 1962) but much more common in children with severe malnutrition.

\section{METHODS}

A series of twenty-five children admitted consecutively to the Tropical Metabolism Research Unit with severe malnutrition according to the Wellcome Classification (Wellcome Trust Working Party, 1970) were studied. There were fourteen boys and eleven girls aged between 7 weeks and 27 months. Normal healthy children, sixteen boys and twelve girls, with ages from 1.5 to 24 months, who attended the Well Baby Clinic of the same hospital, were used as a comparison group. Weight, height, arm circumference and triceps skinfold thickness were measured using standard methods (Jelliffe, 1966) and compared with accepted international reference measurements (Jelliffe, 1966; Cole, 1979). The parent of each child responded to a questionnaire, which was designed to elicit historical information that might have been related to thiamin deficiency. The malnourished children were treated in a standard way during hospitalization as described elsewhere (Jackson \& Golden, 1983). Informed consent was received from the parent or guardian of each child, and the study was approved by the Ethical Committee of the University Hospital of the West Indies.

\section{Samples}

Blood $(1 \mathrm{ml})$ was taken and added to EDTA. The normal children had blood taken on a single occasion and the malnourished children had blood taken (1) on admission before any vitamin supplements had been given $(n 25)$, (2) 2-3 d after the start of high-energy feeding ( $n$ 21), (3) after clinical recovery, i.e. the achievement of an appropriate weightfor-height $(n 17)$. After centrifugation $(2500 \mathrm{~g}$ for $5 \mathrm{~min}$ ) the plasma was removed and an equal volume of saline ( $9 \mathrm{~g}$ sodium chloride / 1) used to reconstitute the erythrocytes. Usually all assays were carried out within $4-6 \mathrm{~h}$ of collection but occasionally the samples were stored at $-20^{\circ}$. All assays were completed within $12 \mathrm{~h}$ of the time of collection. The ETKA was measured by a modification of the micromethod of Dreyfus (1962). The production of sedoheptulose from added ribose-5-phosphate (basic activity) and with exogenous thiamin pyrophosphate (TPP) (stimulated activity) was measured spectrophotometrically against a standard curve. The reproducibility of the standard curve was found to be $\pm 7 \%$. The stimulation of ETKA in the presence of exogenous TPP was expressed as a percentage stimulation (TPP-E\%). The criteria of Brin et al. (1965) were used to determine thiamin status in relation to the TPP-E $\%$, i.e. thiamin status was marginal at $>15 \%$ and deficient at $>30 \%$. The haemoglobin $(\mathrm{Hb})$ concentration in the reconstituted erythrocytes was measured by the cyanmethaemoglobin method and used as a reference to express ETKA.

\section{Statistical analysis}

Differences in thiamin status were compared using paired and unpaired Student's $t$ test for within and betwen group comparisons respectively. Yates' chi-squared or Fisher's exact tests were used for the differences in possible correlates of thiamin status, as appropriate.

\section{RESULTS}

The sexes were similarly represented in the two groups of children although the normal children tended to be younger than the malnourished children (Table 1). The malnourished children were both severely stunted, height-for-age $87 \%$, and severely wasted, weightfor-height $67 \%$ (Waterlow, 1976). 
Table 1. The age and anthropometry of the normal children and the malnourished Jamaican children at the time of admission

(Values are means and standard deviations; heights were not measured in the normal children)

\begin{tabular}{|c|c|c|c|c|}
\hline & \multicolumn{2}{|c|}{ Normal } & \multicolumn{2}{|c|}{ Malnourished } \\
\hline & Mean & SD & Mean & SD \\
\hline Age (months) & 11 & 6 & 12 & 5 \\
\hline No. & 28 & - & 25 & - \\
\hline Height-for-age $(\%)$ & ND & - & 87 & 6 \\
\hline Weight-for-age $(\%)$ & 105 & 16 & 55 & 11 \\
\hline Weight-for-height $(\%)$ & ND & - & 67 & 9 \\
\hline
\end{tabular}

ND, not determined.

Table 2. Erythrocyte transketolase (EC 2.2.1.1) activity measured as sedoheptulose production rate, with and without exogenous thiamin pyrophosphate and the percentage stimulation $(T P P-E \%)$ with thiamin pyrophosphate in normal children, and malnourished children on admission, during and after recovery

(Values are means and standard deviations)

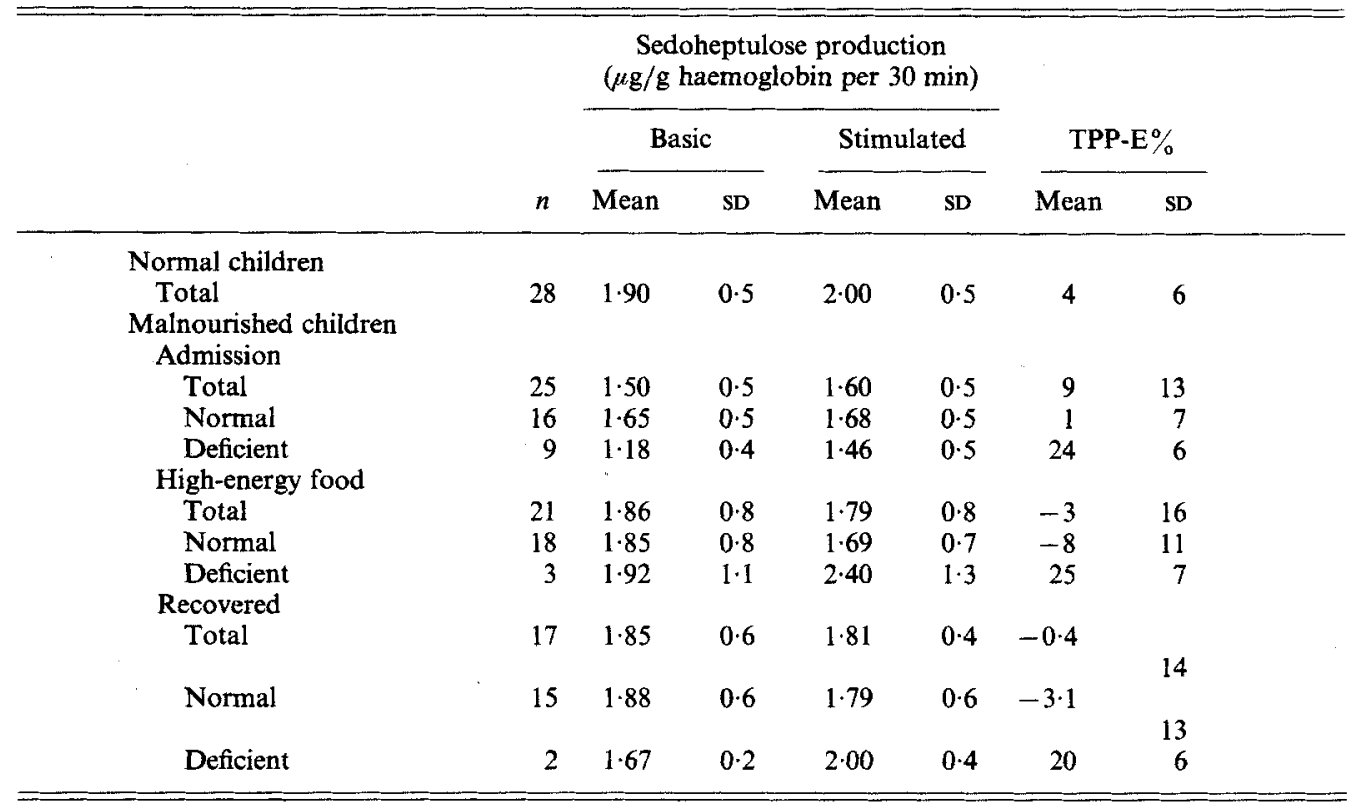

Table 2 shows that the ETKA ( $\mu$ g sedoheptulose/g Hb per $30 \mathrm{~min}$ ) in the normal children was 1.9 basal and 2.0 stimulated, giving a TPP-E $\%$ of 4 . In malnourished children the respective values were 1.5 and 1.6 with a TPP-E $\%$ of 9 . Both basic and stimulated activities were significantly reduced in the malnourished group $(P<0.01)$. Using the criteria of Brin et al. (1965), two of the normal children $(7 \%)$ manifested marginal thiamin deficiency, compared with seven marginal and two deficient $(36 \%)$ of the malnourished group; a significant difference $(P<0.05)$. There was no obvious relation between thiamin status and age.

The thiamin status of the malnourished children in relation to the type of malnutrition 


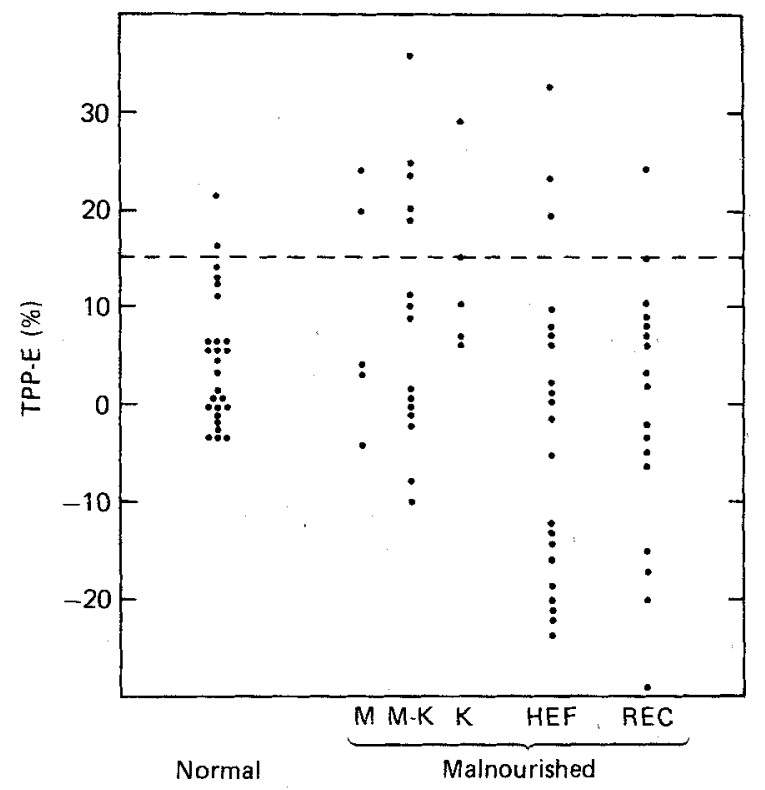

Fig. 1. The stimulation of sedoheptulose production brought about by exogenous thiamin pyrophosphate expressed as a percentage increase (TPP-E\%) in erythrocyte transketolase (EC 2.2.1.1) activity, in normal and malnourished children with either marasmus (M), marasmic kwashiorkor (M-K) or kwashiorkor $(\mathrm{K})$ on admission, after the introduction of high-energy feeds (HEF) and at clinical recovery (REC). (--), The upper limit of normal (Brin et al. 1965). The increase in negative values as recovery progressed may reflect an inhibitory effect of thiamin in vitro (Warnock, 1970).

(Wellcome Trust Working Party, 1970) is shown in Fig. 1. Two of the five children with marasmus, two of the five with kwashiorkor and five of the fifteen with marasmic kwashiorkor showed evidence of deficiency. The basic and stimulated enzyme activities were similar among the three types of malnutrition. Fig. 1 shows the change in TPP-E $\%$ in the malnourished children with recovery. An inadequate thiamin status was noted in nine of the twenty-five children on admission, three of the twenty-one during high-energy feeding and two of the seventeen at recovery.

The basic activity increased significantly from admission ( $80 \%$ of normal) to the time of high-energy feeding ( $90 \%$ of normal). There was no further increase at recovery, when it was still significantly reduced compared with that of the normal children (Table 2). The thiamin-deficient children on admission, in particular, had a marked reduction in basic activity which was not seen in the thiamin-deficient children during or after recovery.

No significant association was found in the malnourished children between thiamin status on admission and age, sex, history of breast-feeding, clinical presentation or anthropometry.

During the study period five children died, between 2 and $16 \mathrm{~d}$ after admission. Four had marasmic kwashiorkor and one kwashiorkor. Four died while receiving a maintenance intake and one after the introduction of high-energy food. The TPP-E $\%$ was within the normal range for all these children on admission. In two children a repeat assay within $24 \mathrm{~h}$ of death showed marginal and severe deficiency (TPP-E $\%, 21$ and $103 \%$ respectively).

The thiamin intake of all the children during the entire period of hospitalization was adequate with an overall mean of $1.37 \mathrm{mg} / 4148 \mathrm{~kJ}(1000 \mathrm{kcal})$. 
DISCUSSION

To our knowledge, this is the first time that ETKA has been used to assess thiamin status in childhood malnutrition, or in a normal population in the Caribbean. In $7 \%$ of normal children the TPP-E \% was outside the accepted range. This contrasts markedly with the finding of thiamin deficiency in $29 \%$ of children in the eastern Caribbean, based on the urinary excretion of thiamin (ICNND, 1962). In North American children, thiamin deficiency was found in 9\% (Morse et al. 1965) and 3\% (Dibble et al. 1965) using ETKA, whereas it was $54 \%$ in Ghanaian children (Neumann et al. 1979) and absent in Nigerian children (Laditan \& Ette, 1983).

Scrimshaw \& Behar (1961) reported normal thiamin levels for malnourished children in Guatemala whereas, in Thailand, urinary excretion tests suggestive of thiamin deficiency were found in marasmus $(9 \%)$, kwashiorkor $(9 \%)$ but not in marasmic kwashiorkor (Thanangkul, 1975). In comparison, our finding of thiamin deficiency in marasmus $(40 \%)$, kwashiorkor $(40 \%)$ and marasmic kwashiorkor $(33 \%)$ appears very high, but is comparable to the $43 \%$ of severely-malnourished children in Ghana (Neumann et al. 1979) and is much less than the report of thiamin deficiency in $84 \%$ of malnourished children from Nigeria (Laditan \& Ette, 1983).

The differences may be explained in part on a methodological basis, given that the urinary excretion of thiamin and the serum concentration of thiamin may not be sensitive indicators of thiamin status (Sauberlich, 1967). We were careful to ensure that our initial measurements were taken on admission before any vitamin supplements had been given. Thiamin status reverted to normal fairly rapidly with the dietary management. However, the differences seen, using the same method, between West Africa and the Caribbean probably represent real geographical variation in the pattern of disease.

It is important to note that thiamin deficiency was as common in oedematous as in non-oedematous malnutrition, making it impossible to draw a simple relation between thiamin deficiency and disturbances in fluid balance and distribution. None of the children manifested the syndrome described by Patrick (1977) after the introduction of high-energy foods, and this may reflect a cautious approach to increasing the energy intake, since this danger has been appreciated. We were unable to show an association with any factor that might have been of use in predicting or identifying the presence of thiamin deficiency. Breast-feeding practices and dietary histories did not help to discriminate, nor did any aspect of the clinical history or the physical signs on admission. This underlines once more the difficulties of associating any one of the clinical features of severe malnutrition to a specific nutrient deficiency (Landman \& Jackson, 1980). Our experience is similar to that of others (Sauberlich, 1967; Neumann et al. 1979; Laditan \& Ette, 1983) in that the clinical features of thiamin deficiency fall far short of specificity.

The time-interval between the first and second blood samples in the malnourished children was of the order of 1 week. During this period they had received a diet providing a maintenance intake of energy and protein with supplements of minerals and vitamins. This was sufficient to correct the thiamin status of all who had been deficient on admission. The children found to be deficient during and after recovery all had a normal thiamin status on admission. Repeat analysis in these children confirmed the abnormal TPP-E \%, which was resistant to therapeutic doses of thiamin. As Table 2 shows, in these children the basic activity was comparable to that of the other children at a similar stage of recovery, and exogenous TPP resulted in relatively high levels of stimulated activity. This is in contrast to many of the children (Fig. 1) in whom there was an increasing tendency towards a negative value for TPP-E $\%$ as recovery progressed. The basic activity, $80 \%$ of normal on admission, rose to $90 \%$ of normal on high-energy feeding, but was still significantly reduced 
at recovery compared with that of the normal children. These changes have been recognized but the explanation is not clear. It has been postulated that mechanisms inhibiting ETKA in vitro may be operative (Warnock, 1970): a shift in equilibrium of the reactions brought about by added thiamin could account for a negative TPP-E \%. In studies on rats (Brin et al. 1960) exogenous thiamin restored ETKA to normal in the early stages of deficiency but, as the deficiency became more severe, restoration of the system became less complete, suggesting a gradual depletion of both coenzyme and apoenzyme. It may be that the depletion of apoenzyme represents a limitation of synthesis during erythropoiesis, and that the changes in basic activity during recovery represent, in part, the production of new erythrocytes with normal enzyme levels mixed with older cells formed during the period of malnutrition which contain a limited amount of transketolase. If this were so then one would expect the basic activity to remain relatively low until the older cells had been removed from the circulation.

The five children who died all had a normal TPP-E \% on admission. Clinically they were the sickest children, with extensive oedema and marked anaemia. We were surprised to find a preterminal deterioration in biochemical thiamin status in two children, as all had received an adequate thiamin intake. It is not possible to say to what extent, if at all, thiamin deficiency contributed to their demise. However, it is interesting that in the recovering and recovered children with an abnormal TPP-E $\%$, the administration of therapeutic doses of thiamin intravenously or orally failed to bring the TPP-E\% into the normal range. We would speculate that there may be situations in which there is a metabolic block to the function of thiamin which, even if present in adequate amounts extracellularly, is unable to exert its effect intracellularly.

Our results lead us to conclude that thiamin deficiency is not found as commonly in the Jamaican child population as we had expected from earlier studies. This may be a reflection of the extensive use of fortified formulas or the generous use of tonics. However, thiamin deficiency is far more common in severe malnutrition than had been previously recognized. We could not link thiamin deficiency with any specific feature of the clinical syndrome but would recommend that care be taken to ensure that all children with severe malnutrition receive an adequate intake of thiamin.

This work was conducted while B.H. was a Fellow of the United Nations University and was supported in part by the Wellcome Trust. The authors are grateful to Dr B. Golden for helping with the collection of samples and the nurses of TMRU for their care of the patients.

\section{REFERENCES}

Brin, M., Dibble, M. V., Peel, A., McMullen, E., Bourquin, A. \& Chen, N. (1965). American Journal of Clinical Nutrition 17, 240-258.

Brin, M., Shohet, S. S. \& Davidson, C. S. (1958). Journal of Biological Chemistry 230, 319-326.

Brin, M., Thai, M., Ostashever, A. S. \& Kalnisky, H. (1960). Journal of Nutrition 71, 273-281.

Cole, J. J. (1979). Annals of Human Biology 6, 249-268.

Dibble, M. V., Brin, M., McMullen, E., Peel, A. \& Chen, N. (1965). American Journal of Clinical Nutrition 17, 218-239.

Dreyfus, P. M. (1962). New England Journal of Medicine 267, 396-398.

Interdepartmental Committee on Nutrition for National Defense (1960). Nutrition Surveys: Chile, pp. $189-222$. Washington DC: A.I.D.

Interdepartmental Committee on Nutrition for National Defense (1962). Nutrition Surveys: The West Indies of Trinidad and Tobago, St Lucia, St Christopher, Nevis and Anguilla, pp. 98-106. Washington DC: A.I.D.

Interdepartmental Committee on Nutrition for National Defense (1963a). Nutrition Surveys: Union of Burma, pp. 91-92. Washington DC: A.I.D.

Interdepartmental Committee on Nutrition for National Defense (1963b). Nutrition Surveys: Ethiopia, pp. $67-84$. Washington DC: A.I.D. 
Jackson, A. A. \& Golden, M. H. N. (1983). In Oxford Textbook of Medicine, pp. 8.12-8.21 [D. J. Weatherall, J. G. G. Ledingham and D. A. Warrell, editors]. Oxford: University Press.

Jelliffe, D. B. (1966). The Assessment of the Nutritional Status of the Community, World Health Organization Monograph Series no. 53, pp. 50-78. Geneva: WHO.

Laditan, A. A. O. \& Ette, S. I. (1983). Journal of Tropical Medicine and Hygiene 86, 85-87.

Landman, J. \& Jackson, A. A. (1980). West Indian Medical Journal 29, 229-237.

Morse, E. H., Merrow, S. D. \& Clark, R. F. (1965). American Journal of Clinical Nutrition 17, $211-217$.

Neumann, C. J., Swenseid, M. E., Jacob, M., Stiehm, E. R. \& Dirige, O. V. (1979). American Journal of Clinical Nutrition 32, 99-104.

Patrick, J. (1977). British Medical Journal i, 1051-1054.

Sauberlich, H. E. (1967). American Journal of Clinical Nutrition 20, 528-542.

Scrimshaw, N. S. \& Behar, M. (1961). Science 133, 2039-2047.

Thanangkul, O. (1975). In Protein Calorie Malnutrition, pp. 150-152. [R. E. Olson, editor]. New York and London: Academic Press.

Warnock, C. G. (1970). Journal of Nutrition 100, 1057-1062.

Waterlow, J. C. (1976). In Nutrition in Preventive Medicine, World Health Organization Monograph Series no. 62, pp. 530-555 [G. H. Beaton and J. M. Bengoa, editors]. Geneva: WHO.

Wellcome Trust Working Party (1970). Lancet ii, 302-303. 